\section{Mirtha Dermisache : L'écriture autre, à elle-même}

Florent Fajole *

Résumé : Mirtha Dermisache (Buenos Aires, 1940-2012) est l'auteure d'écritures illisibles développées dans des formats d'expression et de communication occidentaux auxquelles elle attribua d'emblée et systématiquement une finalité éditoriale. "Mon travail, affirmet-elle, ne veut rien dire. Il ne prend de la valeur que lorsqu'un individu s'exprime à travers lui'. L'auteur de cet article présente la manière dont il a entrepris, en tant qu'éditeur, de relever le défi de cette invitation afin de mettre en évidence leurs incidences sur le dispositif de l'œuvre. La question du temps apparaît alors comme centrale, à la fois dans le travail d'écriture, car ses formes évoluent constamment, et dans la phase éditoriale envisagée comme un processus d'actualisation.

Mots-clés : Mirtha Dermisache - Livre d'artiste - Dispositif éditorial - Écriture illisible Process art.

[Résumés en espagnol, anglais et portugais sur les pages 96-97]

${ }^{(*)}$ Est éditeur de livres et de publications d'artistes. Depuis 2003, il publie régulièrement le travail de l'artiste argentine Mirtha Dermisache (1940-2012) avec qui il a également réalisé plusieurs "dispositifs éditoriaux" à Buenos Aires et en Europe. Il collabore régulièrement avec la galerie Henrique Faria -Buenos Aires et New York- pour la diffusion de son œuvre. En 2017, il a créé Immanences éditions, en association avec Anne-Lou Buzot et Nicolas Peyre. Il est également bibliothécaire et enseigne à l'Ecole nationale supérieure LouisLumière (Saint-Denis, France). Florent Fajole, Immanences éditions, 6 rue des Minimes, 02200 Soissons France. (Voir CV en espagnol sur p. 96)

Aucun code ne produit le nivellement ou l'homogénéisation, le point de fixation qui stabilise la lecture. Les écritures de l'artiste argentine Mirtha Dermisache (1940-2012) sont insaisissables, leur trébuchement formel n'est qu'un leurre. Que le trait se contienne dans son exercice (Voir la Figure 1) ou se laisse surprendre par ses effluves (Voir la Figure 2), comme chez Henri Michaux ${ }^{1}$, l'écriture est autre à elle-même, douée d'“autreté". Elle se prend dans les mailles d'un filet plus ou moins à sa mesure et se déprend déjà, rapidement étrangère ; et si elle hésite ou se reprend, c'est qu'un avenir différent se prépare. Ce qui serait normalement rejeté dans l'ordre sémantique, parce que ne livrant pas le signe ni le sens escompté, devient ici la condition même de leur existence. 


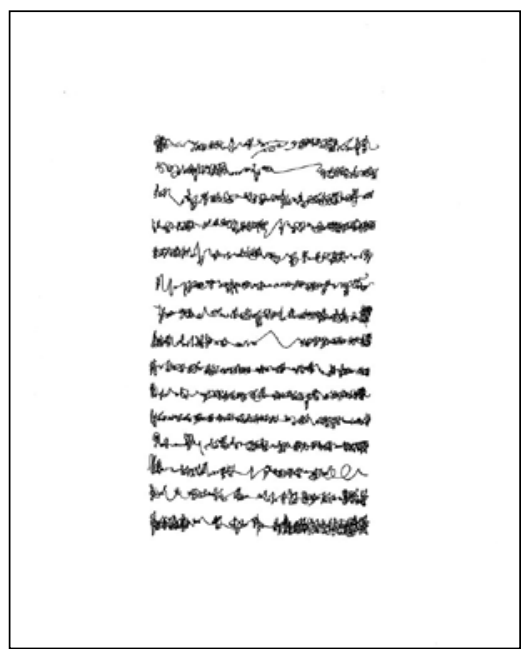

Figure 1.

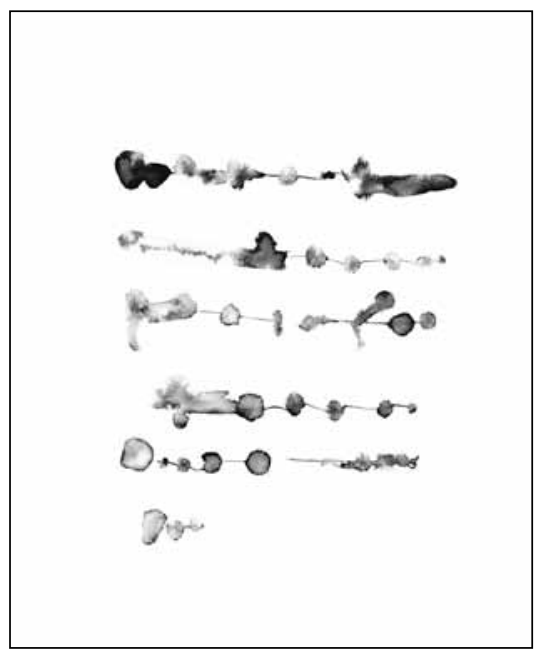

Figure 2.

Figure 1. Libro $N^{\circ} 8,1970$ (Dermisache, 2003). Buenos Aires, Marseille, Nîmes. Xul, Mobil-Home, Manglar. 23 x 28 cm, 700 exemplaires, offset. Figure 2. Extrait de Cinco Textos, 1974 (Dermisache, 2014). Nîmes, Paris, New York, Buenos Aires. Florent Fajole éditeur, Henrique Faria Fine Art, 11 x 7 galería, Fundación Espigas. $23 \times 28 \mathrm{~cm}, 8$ exemplaires comprenant 48 tirages au palladium et 12 tirages au chromopalladium.

Toutefois, à la différence de son aîné, Mirtha Dermisache n'en réfère pas à l'art pariétal ni au primat des idéogrammes chinois. C'est dans notre culture, dans les conditions mêmes de sa technicité et de son formalisme, que des libertés nouvelles, oubliées ou ignorées sont explorées. Il s'agit bien là de recouvrer et d'exprimer une part maudite dans le monde qui est le nôtre, comme pour Christian Dotremont, mais de façon asymétrique. Chez lui, en effet, "la main écrit quand même", remarque Luc Richir, elle "fait le vide de toute préméditation formelle" (Richir, 1974, n/p.) sans renoncer à la langue des mots, tandis que celle de Mirtha Dermisache s'aventure au-delà du sens et de "l'alibi référentiel" (Barthes, 2000, p. 43), dans le seul cadre qui puisse alors lui permettre de continuer à écrire, celui de nos mises en forme.

L'artiste argentine installe ainsi dans le cour même de l'écriture "l'angle dynamique du conflit" (Didi-Huberman, 2009, p. 86), pour reprendre une expression employée par Georges Didi-Huberman dans un autre contexte. L'écriture en tant que dispositif est interrogée "comme un choc des hétérogénéités" (Didi-Huberman, 2009, p. 86), un espace de travail caractérisé par les tensions internes entre un moule qui distribue et organise et le geste qui libère et produit des "formes qui ne sont pas réductibles à quelque modèle que ce soit" (Richir, 1974, n/p). Par conséquent, seuls les formats d'expression et de commu- 
nication qui contiennent les écritures sont pour ainsi dire lisibles ${ }^{2}$; et cet aspect, souvent inaperçu ou déconsidéré, nécessite que l'on s'y attarde quelque peu.

Dans la première lettre qu'il adressa à Mirtha Dermisache, le 28 mars 1971, et qui sert depuis longtemps d'incipit théorique, Roland Barthes écrit :

Vous avez su produire un certain nombre de formes, ni figuratives, ni abstraites, que l'on pourrait ranger sous le nom d'écriture illisible -ce qui revient à proposer à vos lecteurs, non point les messages ni même les formes contingentes de l'expression, mais l'idée, l'essence d'écriture. Rien n'est plus difficile que de produire une essence, c'est-à-dire une forme qui ne renvoie qu'à son nom ; des artistes japonais n'ont-ils pas mis toute une vie à savoir tracer un cercle qui ne renvoie qu'à l'idée de cercle ? Votre travail s'apparente à une telle exigence (Barthes, 2004, p. 11).

Si l'on ne peut qu'être en accord avec le sémiologue français au sujet des "messages", il en va différemment des "formes contingentes" car non seulement celles-ci contraignent les tracés à un certain mode d'organisation et, pour cette raison, à une certaine "prise de forme", mais elles donnent également leurs titres aux œuvres, attribuant de ce fait une dimension signifiante à l'emploi même de ces formats pour les identifier, c'est-à-dire pour leur assigner une identité. Ainsi, Mirtha Dermisache a notamment produit des Artículos, des Cartas, un Diario, des Libros, des Newsletters, des Postales -cartes postales-, des Reportajes, des Texto, etc. Dans La Photographie. Entre document et art contemporain (Rouillé, 2005), André Rouillé fait un constat similaire à propos de l'auteur de La chambre claire, à qui il reproche de négliger l'écriture photographique. Barthes, dit Rouillé dans un entretien accordé à l'occasion de la parution de son ouvrage, ne voit que la chose photographiée, imprimée sur les sels d'argent.

Pour lui, l'image est transparente. C'est un document. Mais la photographie n'est pas seulement un reflet des choses, un enregistreur, c'est surtout une reconstruction de la réalité. C'est ce que Barthes ne dit jamais. Il y a toujours un rapport dialogique avec le monde et les choses, une composition, une esthétique. (Rouillé, 2005, n/p)

Et les "formes contingentes" en font partie, elles propulsent l'écriture dans une réalité matérielle et physique et, ce faisant, l'inscrivent dans un temps culturel, qui peut, assurément, si on le souhaite, ne pas être prisonnier du "repérage"3.

Cette part de lisibilité explique également que Mirtha Dermisache ait d'emblée et systématiquement conçu son œuvre dans une perspective éditoriale, avec des moyens de reproductibilité de masse même si cela n'exclut nullement le recours à un large éventail de procédés. La réalisation d'un travail "original", produit à la plume, au rotring, au feutre ou sans autre appareillage que la main, n'a d'autre finalité que d'exister sous la forme ultérieure d'une publication.

Mirtha Dermisache est ainsi, dans un large contexte de la poésie expérimentale et de l'art contemporain concerné par les problématiques de l'écriture et du langage, une artiste 
pionnière dans le domaine des publications d'artistes en Amérique du sud, au même titre que les frères Augusto et Haroldo de Campos, Ulises Carrión, Moacy Cirne, Guillermo Deisler, Wlademir Dias-Pino, Carlos Ginzburg, Jorge de Luján Gutiérrez, Clemente Padín, Luis Pazos, Décio Pignatari, Julio Plaza, Álvaro et Neide de Sá, Edgardo-Antonio Vigo, ou encore Pedro Xisto, sans oublier Joaquín Torres García sans doute le tout premier d'entreeux dans les années 1930.

Dans un article essentiel pour comprendre la démarche et l'œuvre de Mirtha Dermisache, publié en 1970, Edgardo Cozarinsky, alors jeune journaliste, relate l'intervention de Jorge Romero Brest, critique et curateur influent de la capitale argentine, auprès de la maison d'édition Paidós, qui proposa de publier un choix d'écritures issu du premier livre réalisé par l'artiste en 1967 sous la forme d'un portfolio (Cozarinsky, 1970, p. 51). Elle refusa car, pour elle, son livre ne pouvait être édité que comme tel et dans sa totalité, soit cinq cent pages. Si l'aventure échoua, de nouvelles opportunités se présentèrent à l'invitation de Jorge Glusberg, qui venait de créer le Centro de Arte y Comunicación (CAyC).

La première publication fut imprimée en offset en 1971 pour l'exposition "Arte de Sistemas", au Museo de Arte Moderno de Buenos Aires. Il s'agit de l'une des nombreuses Cartas réalisées l'année précédente. En 1972, à l'occasion de “Arte de Sistemas II", paraissait à plus de mille exemplaires Diario $N^{\circ} 1$, Año 1 (Voir la Figure 3) ; une œuvre considérée pendant longtemps comme un condensé du travail de Mirtha Dermisache et la seule publication à avoir été rééditée à l'identique, dont à deux reprises par l'artiste en 1973 et 1995. Par sa structure, le journal se prête particulièrement bien à sa démarche puisque la division de l'espace sous la forme d'articles et de rubriques et leur organisation en colonnes ou en cases permettent d'accueillir des développements graphiques indépendants tout en jouant de hauteurs, de largeurs, de rythmes et d'intensités variés.

Le CAyC publia également Libro $N^{\circ} 1,1969$, sous le simple titre de "dermisache", à l'occasion de l'exposition Kunstsystemen in Latijns-Amerika à l'Internationaal Cultureel Centrum d'Anvers en 1974. Cet événement fut à l'origine de la rencontre décisive avec Guy Schraenen (2017, pp. 33-47), qui publiera plusieurs travaux de l'artiste entre 1975 et 1978, et avec Marc Dachy, qui l'invita à participer au deuxième numéro de la revue Luna Park en 1976 -consacré aux graphies d'artistes et d'écrivains- et à deux expositions, l'une intitulée "Graphies" au Musée provisoire d'Art Moderne de Bruxelles en 1977 et l'autre "Écritures, Graphies, Notations, Typographies" à la Fondation Nationale des Arts Graphiques et Plastiques, à Paris, en 1980.

Dans le reportage d'Edgardo Cozarinsky, Mirtha Dermisache envisage l'édition de son premier livre de façon précise. L'intérieur ne contient que le travail de l'artiste. Les pages de titre et de colophon sont présentes mais restent vierges. Les couvertures elles-mêmes demeurent tout aussi blanches. Les informations éditoriales sont réunies sur l'un des rabats avec la mention “à jeter". En d'autres termes, seule une maigre concession à l'économie éditoriale traditionnelle est consentie, celle de tolérer l'identification temporaire du livre. Enfin, aucune pagination n'est imprimée.

Les raisons d'une telle radicalité s'imposent d'elles-mêmes. À l'instar du célèbre Coup de dés de Mallarmé ou de la poésie concrète des années 1950, les zones laissées en blanc sont tout aussi signifiantes que les écritures. Intégrer un quelconque signe conventionnel reviendrait à replacer la dimension sémantique dans un espace qui précisément s'en affranchit. C'est 


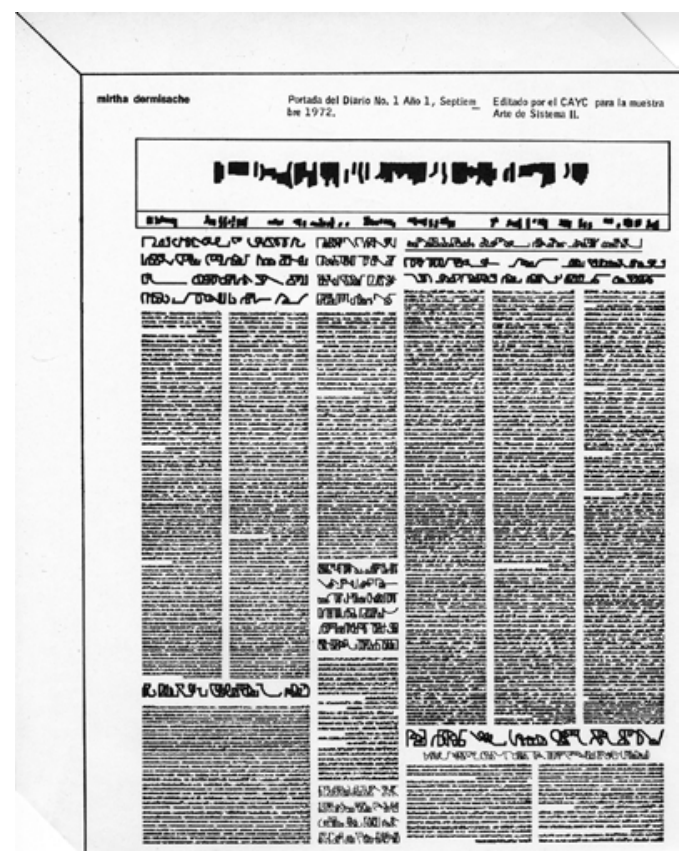

Figure 3. "Feuille volante extraite du catalogue de l'exposition. Arte de Sistemas II". Annonçant à la fois la participation de Mirtha Dermisache et l'édition de Diario $N^{\circ} 1$ Año 1. (1972). Buenos Aires, Centro de Arte y Comunicación. US Letter $(21,6 \mathrm{x}$ $27,9 \mathrm{~cm}$.), tirage inconnu, offset.

aussi la raison pour laquelle, Mirtha Dermisache se limite à indiquer les formats d'expression ou de communication dans les titres de ses publications et que ceux-ci ne comportent aucune connotation d'ordre psychologique voire politique, comme chez Bernard Réquichot (1976) ou souvent chez León Ferrari (2002). Il y a une autre raison encore, c'est le goût du retrait, ou plus précisément le goût de l'autre, discret mais constant, qui situe le lecteur au centre du dispositif de l'œuvre et le convertit en l'un de ses acteurs ; et qui faisait Mirtha Dermisache préférer le silence à propos de son travail (Dachy, 1978, p. 15), à l'instar de Roland Barthes qui, quant à lui, déclarait au sujet de ses propres écritures : "Si mes graphies sont illisibles, c'est bien précisément pour dire non au commentaire” (Barthes, 1976, $\mathrm{n} / \mathrm{p})$. S'ils semblent partager la même attitude, il n'en est rien. Alors que Roland Barthes cherchait, par la pratique de l'écriture illisible, à taire tout discours pour accéder à l'essence d'écriture, "une forme qui ne renvoie qu'à son nom", Mirtha Dermisache adopte cette attitude pour laisser libre champ à ses lecteurs. Selon elle, la plus belle des reconnaissances, au-delà d'être comprise, était que son travail puisse donner matière à expression.

L'article d'Edgardo Cozarinsky pourra donc être envisagé comme un manifeste -l'un des rares- déguisé sous les traits d'un reportage -la lecture proposée par son interlocuteur-, ce que confirme la déclaration de l'artiste que l'auteur reprend à son compte en guise de conclusion : 
Lo mío no quiere decir nada. Unicamente cobra valor cuando el individuo que lo toma se expresa a través de él (mon travail ne veut rien dire. Il ne prend de la valeur que lorsqu'un individu s'exprime à travers lui) (Cozarinsky, 1970, p. 51).

En effet, une autre manière de faire vivre l'hétérogénéité au sein de ce travail, de prolonger l'expérience de l'écriture, autre à elle-même, consiste à conférer à la lecture la dimension d'un acte(s) de pensée(s) et/ou de création. La lecture constitue ainsi le deuxième temps de l'œuvre, synonyme de créativité et d'expression. Par exemple, Mirtha Dermisache invitait quiconque aurait un exemplaire de Diario $N^{\circ} 1$, Año 1 entre les mains à investir les cases d'une courte bande dessinée, figurant en page 7, au sein desquelles l'artiste avait laissé un espace vacant. Il en fut de même pour l'ensemble des Historietas -bandes dessinées- qu'elle a produites à la même époque, vers 1972. Certaines cases furent même laissées entièrement à la disposition du lecteur.

C'est une posture que l'on placera en regard d'une part du Taller de Acciones Creativas qu'elle fonda en 1973 et d'autre part des Jornadas del Color y de la Forma qu'elle coordonna entre 1975 et $1981^{4}$. Tout individu était convié à œuvrer librement dans le cadre collectif d'un "arte por sumatoria", dans un contexte de l'art contemporain où se développait une diversité de pratiques collaboratives qui remirent en cause le principe de l'œuvre d'art uniforme et délimitée dans l'espace et dans le temps. De nombreux artistes latinoaméricains contribuèrent au développement de ces pratiques, notamment au sein des réseaux de la poésie expérimentale et du Mail Art, dont Jorge Caraballo, Carlos Ginzburg, Graciela Gutiérrez Marx, Clemente Padín, Luis Pazos, Juan Carlos Romero, Edgardo-Antonio Vigo, et Horacio Zabala, ont été les figures rioplatenses majeures, et auxquels Mirtha Dermisache fut indirectement associée par le biais du CAyC $\mathrm{C}^{5}$, puis directement par celui de Guy Schraenen et d'Ulises Carrión. Ces artistes pratiquaient une poésie et un art hors de l'objet, dans l'espace public et hors des sentiers usités. Bien souvent, ce qui comptait, c'était “créer l'amorce, le geste initial et se laisser dépasser", écrit Julien Blaine en 1967, car "ce dépassement est riche de je" (Blaine -1-, 1967, n/p), d'étendues, aurait dit Michaux (1963, p. 51), de multiplicités et de singularités ; "l'écriture n'étant pas ce résultat mais les gestes qui l'ont précédé et suivi" (Blaine -2-, 1967, n/p). Il s'agit bien de la même attitude à laquelle s'est toujours résolue Mirtha Dermisache.

Nous retrouvons ici la dimension du dispositif chère à Georges Didi-Huberman (2009), si l'on veut bien considérer chacune de ces pratiques comme un "choc des hétérogénéités", et dans la perspective offerte par François Albera et Maria Tortajada (2011) pour qui cette même notion de dispositif désigne un agencement qui ne peut être confondu avec un objet ou un appareil, éclairant la différence essentielle entre ce qui se présente comme un assemblage d'éléments hétérogènes et ce que l'on appréhende comme un tout, dont les “organes" apparaissent indissociables. Ainsi, souligne Rémy Bresson, un dispositif est moins "une chose identifiable qu'un système de relations entre différentes choses" (Bresson, 2012). La différence ne se situe donc pas dans la possibilité ou non de faire système -ce que du reste indiquait le titre choisi, "Arte de sistemas", par Jorge Glusberg pour qualifier ce nouveau type d'art dont il réunit diverses formes dans le cadre des activités du CAyCmais dans celle de l'ouvrir, de pénétrer l'œuvre et de l'envisager comme un milieu changeant et multiple. 
L'écriture et l'œuvre dans son ensemble, de la production de "l'original" à son édition, doivent en quelque sorte constamment se dépasser pour vérifier leur niveau et s'étendre au-delà de leur première instance, tout au long d'un processus qui tient compte de l'effet de chaque lecture -et de chaque écriture si l'on donne à ce mot le sens d'une action menée après lecture. L'intervention de l'éditeur n'a pas d'autre objet que d'impulser un trajet dans l'œuvre pour en explorer et en alimenter le devenir.

En 2003, parallèlement à Libro $N^{\circ} 8,1970$, je proposai à Mirtha Dermisache de réaliser une édition limitée d'un livre qu'elle produirait spécialement et lui suggérai d'utiliser le même papier pour l'impression offset que celui qu'elle utiliserait pour écrire, de manière à insérer chaque feuille originale en lieu et place de sa reproduction dans un nombre d'exemplaires correspondants au nombre de ces feuilles. Le livre créé par l'artiste, composé de dix feuilles écrites -douze au total en y ajoutant une feuille de titre et une feuille de colophon vierges-, se trouve disséminé parmi dix exemplaires formant le tirage commercial de cette édition. Dix autres exemplaires, hors commerce, ne comprenant que des feuilles imprimées et vierges complétèrent le tirage. C'est ainsi que nous réalisâmes intégralement Libro $N^{\circ} 1,2003$, en subsumant la production initiale sous son édition ; processus qui est celui d'une œuvre portée au-delà de ses gestes initiaux.

La même année, Olga Martínez invita l'artiste argentine à réaliser sa première exposition individuelle à Buenos Aires, à la galerie El Borde, qu'elle avait créé quelques temps auparavant. Je glissai alors l'idée à Mirtha Dermisache de situer l'enjeu de l'appropriation dans un cadre scénographique car, s'il était évident que l'on devait affirmer le caractère éditorial de l'œuvre, il était également nécessaire qu'elle intègre les protocoles et le système de l'art afin d'y trouver son public -sans quoi, du reste, l'invitation aurait été rejetée. Il n'y avait, par ailleurs, aucune raison de créer des catégories spécifiques de lecteurs; l'un de nos objectifs était à l'inverse de convertir un lieu privé, ce qu'est une galerie d'art, en un espace public. Ces paramètres m'incitèrent alors à imaginer un "dispositif éditorial”, c'est-à-dire un dispositif constituant une édition en tant que telle, composé de douze chaises et de dix tables en bois à la forme géométrique élémentaire, peintes en blanc, réparties et espacées de façon régulière dans une salle rectangulaire de $100 \mathrm{~m}^{2}$ environ. Sur ces tables était placé et offert gratuitement le contenu d'une publication imprimée en offset à 400 exemplaires ayant pour titre Nueve Newsletters \& Un Reportaje (Voir la Figure 4), dix travaux composés chacun d'une seule feuille, datant de 1999 et de 2000. Le dispositif fut réalisé durant le mois d'août 2004 (Voir la Figure 5). Le public était invité à circuler dans le corps de l'installation et pouvait quitter le lieu avec son propre choix éditorial, créant ainsi une instance particulière de l'œuvre par l'acte de la sélection et du-ou des-parcours préalable(s) : une façon concrète d'agir sur le "niveau" de l'œuvre -chaque niveau étant autonome- jusqu'à son épuisement, qui n'est rien d'autre que son accomplissement.

De ce point de vue, l'exemple de Diario $N^{\circ} 1, A \tilde{n} o 1$, au sein duquel le lecteur peut luimême écrire, est une instance comparable au mouvement que Mirtha Dermisache et moi donnâmes ensemble, bien des années après, au processus éditorial. Aussi, l'œuvre ne se défait pas de l'alibi référentiel uniquement sur le plan sémantique, ce principe s'étend aussi à la notion d'autorité, expliquant l'effacement de l'artiste et le primat de la lecture -et par voie de conséquence de l'édition- une fois les originaux produits. Mirtha Dermisache l'avait indiqué à Edgardo Cozarinsky, qui le retranscrit dès le début de son article. De son 


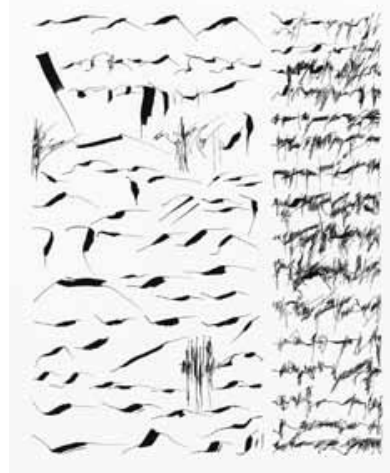

Figure 4.

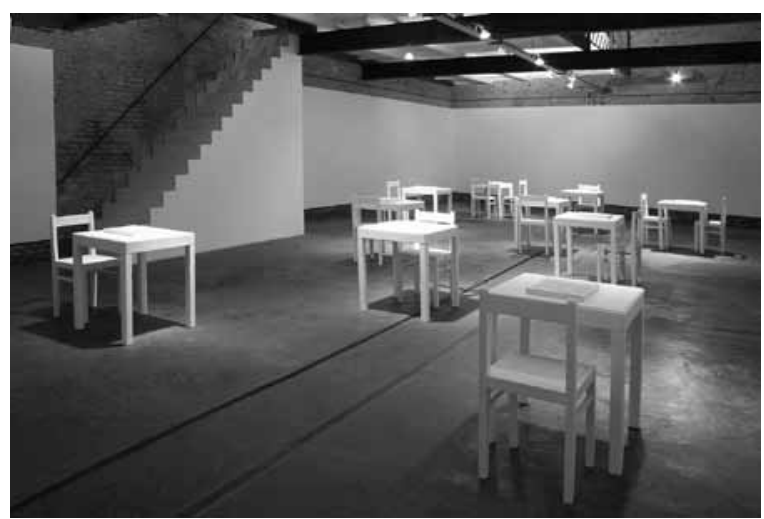

Figure 5.

Figure 4. Extrait de Mirtha Dermisache, 9 Newsletters \& 1 Reportaje (2004). Buenos Aires, Marseille, Nîmes. El Borde, Mobil-home, Manglar. 27,5 x 32,5 cm, 400 exemplaires, offset. Figure 5. "Mirtha Dermisache, Escrituras] : [Múltiples” (2011). Dispositif éditorial réalisé à l'Espacio de Arte contemporáneo El Borde. Buenos Aires, Argentine. Edition et scénographie : Florent Fajole.

premier livre, elle affirmait : "es un producto, lo quiero independiente de mi persona (c'est un produit, je veux qu'il soit indépendant de ma personne)" (Cozarinsky, 1970, p. 51). Cette posture n'a pas varié dans le temps et engage, de quelque façon que ce soit, ceux qui ont édité son œuvre de son vivant. Ainsi, Guy Schraenen a toujours réservé une place de premier ordre aux publications de Mirtha Dermisache dans ses différentes expositions. Ce choix ne s'explique pas seulement par l'amitié qui les lia mais aussi et fondamentalement par le sens même donné à l'édition dans le processus de création. Pour ma part, lorsque je découvris l'article d'Edgardo Cozarinsky en 2003, il m'a semblé tout de suite indispensable d'interroger le dispositif de l'œuvre et d'en saisir les enjeux. Quelques années plus tard, en 2008, 2009, les écrits de Gilles Deleuze et de Félix Guattari sur la lecture et le principe de l'actualisation allaient confirmer le regard que je portais ; et c'est sous les mots d'Anne Sauvagnargues que je trouvai un résumé de cet édifice théorique offrant une véritable résonance à mon propos, avec la précaution qui nous invitera à remplacer "corps sans organes" par "corps désorganisable", "milieu changeant", "œuvre en devenir", ou "oeuvrement" qui me semblent aujourd'hui des notions plus appropriées que le concept forgé par Gilles Deleuze à partir d'Antonin Artaud ; et à défaut de pouvoir employer en français des expressions dérivant d'un verbe capable de désigner ce qui est en jeu, comme cela est possible en anglais, "to process", ou en espagnol, "procesar"6:

L'œuvre cesse de renvoyer à une totalité organique, son corps, sans organes, ne présente pas d'orientation définie ni de sens fixe. L'entrée dans l'œuvre est frag- 
mentaire : c'est nécessairement qu'elle élit un point quelconque dans l'œuvre, point d'entrée qui force un trajet dans l'œuvre. Ce qui est quelconque, non prédéterminé, c'est le point d'entrée critique. On entrera par n'importe quel bout, l'insertion critique n'étant pas quelconque au sens où elle serait indifférente, ni arbitraire, mais bien aléatoire, c'est-à-dire remarquable pour cet événement de lecture. "Aucune entrée n'a de privilège", puisqu'il n'y a plus de totalité hiérarchisant les dimensions et distinguant les extrémités. L'entrée est, “on peut l'espérer, en connexion avec d'autres choses à venir". A l'entrée déterminée comme portail, s'est substituée l'effraction cinétique, qui tient compte de l'effet de la lecture sur l'œuvre, puisque c'est le point choisi qui détermine tel trajet. On trouve là une application de l'actualisation : le point d'entrée agit comme un germe de cristallisation qui prend ou non corps dans le matériau de l'œuvre. Toutes les lectures ne se valent donc pas, mais chacune transforme l'œuvre, sur le mode rhizomatique d'une cartographie (Sauvagnargues, 2009, p. 366).

Depuis la réalisation du premier dispositif éditorial, j'aborde l'édition du travail de Mirtha Dermisache sous trois angles complémentaires. Tout d'abord, la publication des "originaux" à l'identique est la première de mes responsabilités. Cela implique un tirage offset à plusieurs centaines d'exemplaires -400 ou 500 au minimum. Le dispositif éditorial est la forme scénographique de diffusion que je privilégie car il permet de placer librement le lecteur face à de multiples choix sans que le facteur économique n'entre en ligne de compte ou en le réduisant au maximum -le prix d'entrée éventuel pour y accéder. En règle générale, je réalise deux types de tirages complémentaires, l'un pour ce type d'intervention et l'autre pour la commercialisation en dehors de ce cadre. Les dispositifs éditoriaux que Mirtha et moi avons réalisés ensemble à Buenos Aires en 2004 et 2005, à Londres en 2006, à Rome en 2007, et à Saint-Yrieix-la-Perche en 2008 consistaient en premier lieu à créer un contexte de diffusion, de lecture, voire d'appropriation, pour des œuvres éditées à l'identique. Il en fut autrement lors du dernier dispositif réalisé ensemble à Buenos Aires en 2011, où je proposai pour la première fois, parmi d'autres publications disposées sur table, trois variantes d'un même Texto de couleurs, Texto, 1974 (Voir la Figure 6), réalisées au chromopalladium, un procédé combinant un tirage photochimique aux sels de palladium et une impression pigmentaire pour les couleurs ${ }^{7}$. Un tel procédé permet de créer des variations de contraste et de densité à chaque tirage, modifiant certains traits de l'œuvre. Il s'agissait d'employer une technique de reproduction, et dans ce cas de tirage -empruntée au monde de la photographie- comme une technique de création afin de générer de nouvelles instances scripturales. Mirtha Dermisache a utilisé un matériau d'impression comme matériau de création pour la première fois en 1975. Guy Schraenen lui proposa d'employer des feuilles de papier carbone pour transférer des tracés effectués à l'aide d'un instrument doté d'un roulement cranté sur des feuilles de report. Ces feuilles de carbone, que l'on peut considérer comme des matrices, furent ensuite insérées séparément dans les exemplaires du tirage de tête de Cahier $N^{\circ} 1,1975$. En ce qui me concerne, le coût élevé du chromopalladium ne permet d'effectuer qu'un nombre d'épreuves suffisant pour servir à leur tour de matrices éditoriales, comme il en est de l'original au début du processus. 

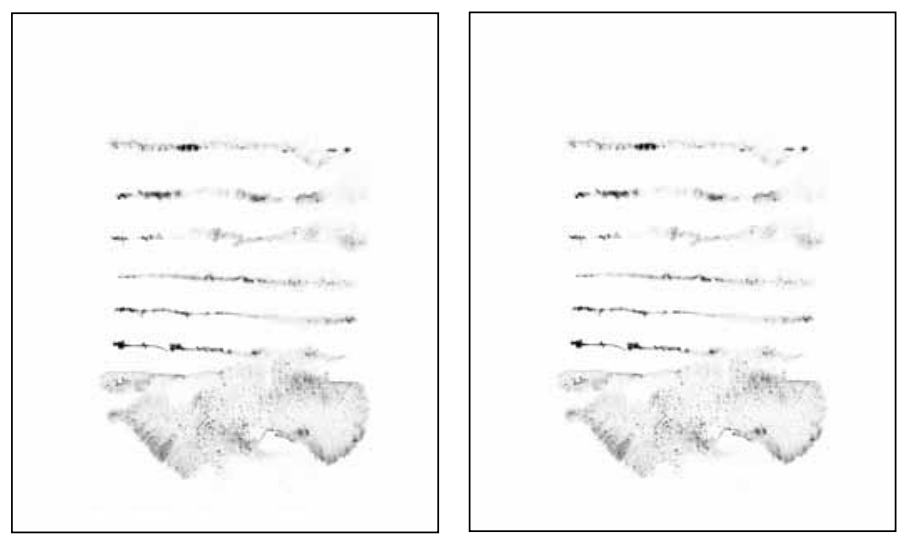

Figure 6.
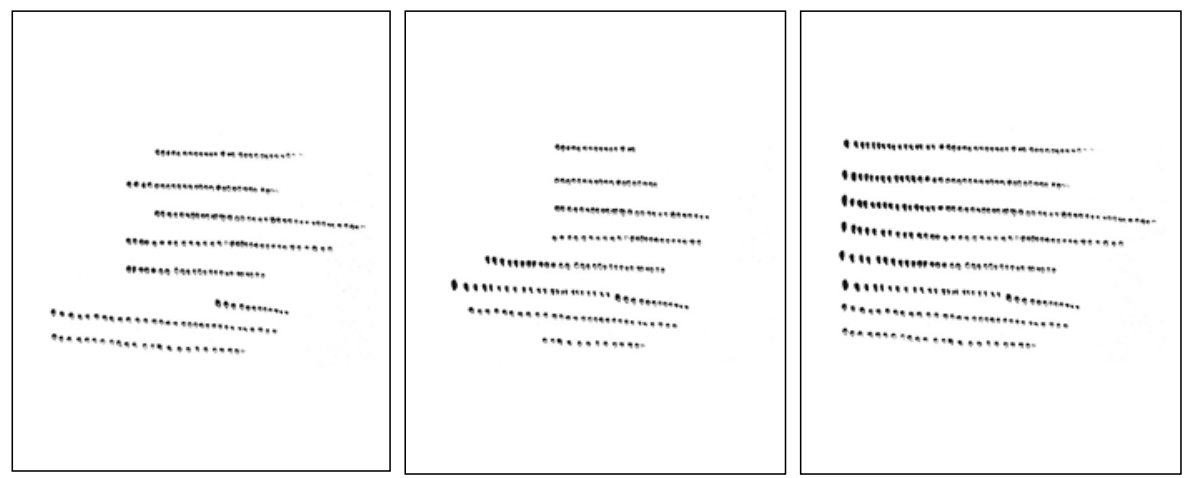

Figure 6.

Figure 6. Trois variantes extraites de Mirtha Dermisache, Texto, 1974 (2011). Nîmes, Paris. Ed. de la Mangrove-Geneviève Chevalier \& Florent Fajole éditeurs-, Atelier Alban Chassagne. 23 x $28 \mathrm{~cm}, 24$ tirages au chromopalladium répartis en 8 exemplaires. Figure 7. Processing Texto, circa 1970 by Mirtha Dermisache (Dermisache, 2017). Soissons, Buenos Aires, New York, Immanences éditions (Anne-Lou Buzot, Florent Fajole \& Nicolas Peyre éditeurs associés), Henrique Faria Fine Art. 23 x 28 cm., 400 exemplaires, offset.

Depuis cette première expérience, j'ai réalisé selon le même principe, avec une gamme de procédés que j'élargis progressivement ${ }^{8}$, en co-édition avec la galerie Henrique Faria, plusieurs publications de différents Textos, dont les contenus seront les matrices de prochaines éditions offset à venir : "des écritures qui agrandissent leurs variations" car, si Christian Dotremont affirmait que "personne n'écrit deux fois mêmement un mot” (Do- 
tremont, 1989, p. 117), il n'en va pas autrement lorsque l'écriture cesse d'évoluer dans un registre sémantique.

Le troisième aspect de mon intervention consiste actuellement, sur un principe analogue à celui de la variante, à proposer des lectures distinctes d'un même original en mettant l'accent sur ses caractéristiques rythmiques. Une première lecture de Texto, circa 1970 (Voir la Figure 7) a donné naissance à trois variations imprimées en offset à 400 exemplaires et diffusées gratuitement sous la forme d'un dispositif éditorial à l'occasion de l'exposition "Mirtha Dermisache: the Otherness of Writings" à la galerie Henrique Faria à New York en décembre 2017. D’autres suivront et pourront donner lieu à des dispositifs éditoriaux de taille variable selon les lieux d'intervention; et les publications seront commercialisées parallèlement.

Ces quelques exemples suffisent à donner la mesure et le sens de la dimension éditoriale dans le travail de Mirtha Dermisache, dont l'objectif, comme l'a remarqué Jérôme Dupeyrat (2012, pp. 227-235), n’a jamais été de réifier les écritures ni les objets ou agencements qui les contiennent mais à l'inverse de placer le regard et l'expression du lecteur au cœur même de ce processus. Le facteur temps, loin de condamner l'œuvre à dépendre de l'artiste qui l'a engendrée, est l'allié de ses développements, de ses actualisations, et de ses multiples instances.

\section{Notes}

1. Geneviève Bonnefoi est l'auteure de superbes textes sur les dessins et les aquarelles d'Henri Michaux. Je me suis inspiré de l'un d'entre eux, publié dans le catalogue de l'exposition organisée par le Stedelijk Museum, à Amsterdam, en 1964, pour écrire ce paragraphe. 2. À ma connaissance, le premier auteur à avoir clairement distingué ce niveau de lisibilité dans l'œuvre de Mirtha Dermisache est Jorge Santiago Perednik (1952-2011), poète, traducteur, critique et éditeur argentin, avec qui Geneviève Chevalier et moi avons fait la connaissance de Mirtha Dermisache en 2002 et co-édité deux de ses livres : Mirtha Dermisache, Libro $N^{\circ}$ 8, 1970, Buenos Aires, Marseille, Nîmes, Xul, Mobil-Home, Manglar, 2003 (Fig. 1) et Mirtha Dermisache, Libro No 1, 2003, Buenos Aires, Marseille, Nîmes, Xul, Mobil-Home, Manglar, 2003. Cf. Jorge Santiago Perednik, "Mirtha Dermisache y la escritura ilegible”. In Intermedial-es, "Mirtha Dermisache”, Nîmes, Manglar, 2005, n/p, publié à l'occasion du dispositif éditorial Mirtha Dermisache, "Lectura pública", Centro Cultural de España, Buenos Aires (2005).

3. Outre Christian Dotremont et Henri Michaux, on pourra comparer cette conception de l'écriture avec celles proposées dans les années 1960, 1970 par des artistes aussi différents qu'Irma Blank, Carlfriedrich Claus, Guy de Cointet, Hanne Darboven, León Ferrari, Bernard Réquichot, ou Mira Schendel.

4. Mirtha Dermisache a énuméré les différents critères de l'approche pédagogique qu'elle menait au sein du Taller de Acciones Creativas dans le catalogue de la dernière exposition réalisée de son vivant à Buenos Aires en 2011. Cf. Annalisa Rimmaudo y Giulia Lamoni, "Entrevista a Mirtha Dermisache". In Mirtha Dermisache. Publicaciones y dispositivos editoriales. (2011, p. 16). Ces critères furent à la base de la méthode employée lors des Jorna- 
das del Color y de la Forma. À ce propos, cf. Lucía Cañada, "Las Jornadas del color y de la forma (1975-1981). El arte como praxis vital”. In Mirtha Dermisache: ¡Porque yo escribo! (2017, pp. 49-63) ; et le texte d'Agustín Pérez Rubio, "Metodología para una libre expresión", ibidem, pp. 65-77.

5. C'est par le biais du CAyC que Clemente Padín et Julien Blaine découvrent le travail de Mirtha Dermisache et publient le même fragment respectivement dans De la représentation à l'action, Les Anartistes, Ventabren, 1975 et dans le $n^{\circ} 1$ de la revue $\operatorname{Doc}(k) s$ en 1976. 6. Il me semble, en effet, que l'on peut parler de "process art" à propos de l'œuvre de Mirtha Dermisache ; et c'est dans cette perspective que je situe mon action.

7. C'est un procédé qu'utilise par exemple la photographe espagnole Isabel Muñoz pour réaliser ses tirages. Cf. le portfolio Amor y Extasis édité par Immanences éditions : http://immanences-editions.com/publications/isabel-mun\%CC\%83oz-amor-y-extasis/ . Consulté le 02/01/2018.

8. Je continue à employer le palladium et le chromopalladium avec Anne-Lou Buzot ; mais également l'impression typographique avec Hannah Harkes (Tallinna Paber, Tallinn, Estonie), et j'envisage prochainement d'expérimenter l'offset lithographique, dans la même ville, et l'héliogravure en France. Après avoir confié l'impression offset à Garbarino Hermanos à Buenos Aires puis à Realtisk à Prague, aujourd'hui c'est Palermo Artes Gráficas (Madrid) qui s'en charge.

\section{Bibliographie}

Albera, F. et Tortajada, M. (2011). “Le dispositif n'existe pas!”. In François Albera et Maria Tortajada (dir.). Ciné-dispositifs : spectacles, cinéma, télévision, littérature. Lausanne, Suisse. L'Âge d'homme.

Bonnefoi, G. (1964). (s/t). In Henri Michaux. Catalogue d'exposition. Amsterdam, Pays-Bas. Stedelijk Museum, 1964.

Barthes, R. (2000). "Variations sur l'écriture". In : Le Plaisir du texte; précédé de Variations sur l'écriture. Paris, France. Ed. du Seuil.

Barthes, R. (2004). "Fragment d'une lettre de Roland Barthes adressée à Mirtha Dermisache, le 28 mars 1971". In : Cahier du Refuge 130, Marseille, France. Centre International de Poésie Marseille.

Barthes, R. (1976). (s/t). In Luna Park, n² 2, Bruxelles, Belgique. Ed. Transédition.

Blaine, J. (1967 -1-) "Je dans le jeu", in Open, n 3" (repris in Julien Blaine, Poésie séméiotique / Poesía semeiótica).

Blaine, J (1967-2-). Breuvages épandus. (s/l). Edition de l'artiste (repris in Julien Blaine, Poésie séméiotique / Poesía semeiótica).

Blaine, J. (2003). Poésie séméiotique / Poesía semeiótica. Buenos Aires, Marseille, Nîmes, Xul, Mobil-Home, Manglar.

Bresson, R. (2012). "Pour une définition de la notion de dispositif”, In Cinémadoc: http:// cinemadoc.hypotheses.org/2621 Consulté le 02/01/2018.

Cañada, L. (2017). "Las Jornadas del color y de la forma (1975-1981). El arte como praxis vital”. In Mirtha Dermisache: ¡Porque yo escribo! Agustín Pérez Rubio, "Metodología 
para una libre expresión”, In Pérez Rubio Agustín (ed.), Mirtha Dermisache: ¡Porque yo escribo! Catalogue d'exposition. Buenos Aires, Argentina. MALBA, Fundación Espigas.

Cozarinsky, E. (1970). “Un grado cero de la escritura”. In Panorama, año VII, n 156. Buenos Aires, Argentina.

Dachy, M (1978). "Gliffpower. Le chant du signe". In Graphies. Catalogue d'exposition. Bruxelles, Belgique. Ed. Musées Royaux des Beaux-Arts, Belgique.

Dermisache, M (1972). Diario $N^{\circ} 1$ Año 1. Buenos Aires, Argentina. Centro de Arte y Comunicación.

Dermisache, M. (2004). Nueve Newsletters \& Un Reportaje, Buenos Aires, Marseille, Nîmes. El Borde, Mobil-Home, Manglar.

Dermisache, M. (2011). Texto, 1974. Nîmes, Paris. Ed. de la Mangrove (Geneviève Chevalier \& Florent Fajole éditeurs), Atelier Alban Chassagne.

Dermisache, M. (2017). Processing Texto, circa 1970 by Mirtha Dermisache, Soissons, Buenos Aires, New York. Immanences éditions (Anne-Lou Buzot, Florent Fajole \& Nicolas Peyre éditeurs associés), Henrique Faria Fine Art.

Didi-Huberman, G (2009). Quand les images prennent position. L'œil de l'histoire 1. Paris, France. Ed. de Minuit.

Dotremont, Ch. (1989). "Sept écritures”. In Abstrates, (s/l), France. Ed. Fata Morgana.

Dupeyrat, J. (2012). Les livres d'artistes entre pratiques alternatives à l'exposition et pratiques d'exposition alternatives, Thèse de doctorat sous la direction de Leszek Brogowski, Rennes, Université Rennes 2. Version consultable sur internet : https://halshs.archives-ouvertes. fr/tel-00772314/document. Consulté le 02/01/2018.

Michaux, H. (1963). Passages (1937-1963). Paris, France. Ed. Gallimard.

Pérez Oramas, L. (2002). León Ferrari and Mira Schendel: Tangled Alphabets. Catalogue d'exposition. New York, USA. The Museum of Modern Art (MoMA).

Pérez Rubio, A. (2017). "Metodología para una libre expresión”, In Pérez Rubio Agustín (ed.), Mirtha Dermisache: ¡Porque yo escribo! Catalogue d'exposition. Buenos Aires, Argentina. MALBA, Fundación Espigas.

Réquichot, B. (1976). Les écrits de Bernard Réquichot. Bruxelles, Belgique. Éd. de la Connaissance.

Richir, L. (1974). “Pour l'exposition de logogrammes de Christian Dotremont en févriermars 1974 à Bruxelles”. Plaquette d'exposition. Galerie Maya, Bruxelles, Belgique. Editions Galerie Maya.

Rimmaudo, A. y Lamoni, G. (2011). “Entrevista a Mirtha Dermisache”. In Fajole Florent (ed.), Mirtha Dermisache. Publicaciones y dispositivos editoriales. Buenos Aires, Argentina. Pabellón de Bellas Artes, Pontificia Universidad Católica Argentina.

Rouillé, A. (2005). La Photographie. Entre document et art contemporain. Paris, France. Ed. Gallimard.

Rouillé, A. (2018). “Quelles images pour quelle réalité? Entretien avec André Rouillé à propos de son ouvrage La Photographie”. Revoirfoto.com : http://www.revoirfoto.com/p/index. php?lg=\&pg=30\&c=7. Consulté le 02/01/2018.

Sauvagnargues, A. (2009). Deleuze. L'empirisme transcendantal. Paris, France. Presses Universitaires de France. 
Schraenen, G. (2017). “Une affaire transatlántico”. In Pérez Rubio Agustín (ed.), Mirtha Dermisache: ¡Porque yo escribo! Catalogue d'exposition. Buenos Aires, Argentina. MALBA, Fundación Espigas.

${ }^{(*)}$ Es editor de libros y publicaciones de artistas. Edita el trabajo de la artista argentina Mirtha Dermisache (1940-2012) desde 2003 y ha sido creador de una serie de "dispositivos editoriales" con ella en Buenos Aires y en Europa. Colabora regularmente con la galería Henrique Faria -Buenos Aires y New York- para la difusión de su obra. En 2017, ha creado la editorial Immanences éditions, junto con Anne-Lou Buzot y Nicolas Peyre. También es bibliotecario y enseña en la Ecole nationale supérieure Louis-Lumière -SaintDenis, France-.

Resumen: Mirtha Dermisache (Buenos Aires, 1940-2012) es una artista que a lo largo de su carrera, ha concentrado su obra sobre la creación y el desarrollo de "escrituras ilegibles" adoptando formatos de expresión y comunicación occidentales, con el fin sistemático de editarlas. "Lo mío, -afirma ella en un reportaje a Edgardo Cozarinsky (1970)-, no quiere decir nada. Unicamente cobra valor cuando el individuo que lo toma se expresa a través de él". El autor de este artículo, editor de Mirtha Dermisache desde 2003, indica como aceptó el reto de esta invitación y destaca sus incidencias sobre el dispositivo de la obra. La cuestión del tiempo aparece como un aspecto clave, tanto en la fase de producción de las escrituras, ya que éstas evolucionan permanentemente, como en la fase editorial considerada como proceso de actualización.

Palabras clave: Mirtha Dermisache - Libro de artista - Dispositivo editorial - Escritura ilegibles - Proceso artístico.

Abstract: Mirtha Dermisache (Buenos Aires, 1940-2012) is the author of a wide production of asemic writings using western expression and communication formats, to which she systematically gave a publishing finality from the very beginning. "My work, she stated, does not mean anything. It only makes sense when someone expresses himself through it". The author of this article presents the way he, as a publisher, undertakes the challenge of such an invitation. The aim is to highlight their implications on the work apparatus or configuration (from the French "dispositif"). Then the question of Time appears as a key aspect of the work, both in the writings because of their permanent movement and in the publishing phase, which is explored as an actualization process.

Keywords: Mirtha Dermisache - Artists' book - Dispositif editorial - Asemic writings Process art.

Resumo: Mirtha Dermisache (Buenos Aires, 1940-2012) é um artista que, ao longo de sua carreira, concentrou seu trabalho na criação e desenvolvimento de "escritos ilegíveis" ado- 
tando formatos de expressão e comunicação ocidentais, com o objetivo sistemático para editá-los. "Minha coisa", ela diz em um relatório para Edgardo Cozarinsky (1970), "não significa nada. Só ganha valor quando o indivíduo que o leva se expressa através dele". O autor deste artigo, editor da Mirtha Dermisache desde 2003, indica como ele aceitou o desafio deste convite e destaca suas incidências no dispositivo do trabalho. A questão do tempo aparece como um aspecto chave, tanto na fase de produção dos escritos, como estes evoluem permanentemente e na fase editorial considerada como o processo de atualização.

Palavras chave: Mirtha Dermisache - Livro dos artistas - Editorial Dispositif - Escritos assépticos - Processo artístico.

[Las traducciones de los abstracts al español, inglés y portugués fueron supervisadas por el autor de cada artículo] 